\title{
Methanolic Root Extract of Rauwolfia serpentina Lowers Atherogenic Dyslipidemia, Arteriosclerosis and Glycosylation Indices in Type 1 Diabetic Mice
}

\author{
Muhammad Bilal Azmi ${ }^{1,2^{*}}$, Shamim A Qureshi ${ }^{1}$, Sumera Rais ${ }^{3}$, Saleha Sultana ${ }^{1}$ \\ ${ }^{1}$ Department of Biochemistry, University of Karachi, Karachi-75270, Pakistan. \\ ${ }^{2}$ Quality Enhancement Cell, Dow University of Health Sciences, Karachi- 74200, Pakistan. \\ ${ }^{3}$ Department of Biochemistry, Federal Urdu University of Arts, Science and Technology, Karachi-75270, Pakistan.
}

\section{ARTICLE INFO \\ Article history: \\ Received on: 22/04/2015 \\ Revised on: 19/05/2015 \\ Accepted on: 06/06/2015 \\ Available online: 28/08/2015}

Key words:

Atherogenic index, cholesterol, triglycerides, Rauwolfia serpentina

\begin{abstract}
Globally recognized antihypertensive phytomedicine Rauwolfia serpentina Benth is also well-known for its diversified ethno-medicinal usefulness. The objective of present study is to evaluate the effect of methanolic root extract (MREt) of $R$. serpentina on atherogenic dyslipidemia, arteriosclerosis and glycosylation indices in alloxan-induced type 1 diabetic mice. After the single intraperitoneal injection of alloxan monohydrate (150 $\mathrm{mg} / \mathrm{kg}$ ), forty two mice were allocated into diabetic control (1 ml distilled water), negative control ( $0.05 \%$ DMSO $1 \mathrm{ml} / \mathrm{kg}$ ), positive control (5 mg/kg glibencalmide) and three test dose groups (MREt $10,30 \& 60 \mathrm{mg} / \mathrm{kg}$ ). Normal control ( $1 \mathrm{ml}$ distilled water) was also used. After 14 days of respective treatments, fasting blood glucose, insulin, haemoglobin $(\mathrm{Hb})$, glycosylated haemoglobin $\left(\mathrm{HbA}_{1 \mathrm{c}}\right)$, triglyceride $(\mathrm{TG})$, total cholesterol (T-CHOL), low density lipoprotein cholesterol (LDL-c), very low density lipoprotein cholesterol (VLDL-c), and high density lipoprotein cholesterol (HDL-c) levels were determined. Wet organ weight, relative weight, percent change in glycosylation and non-HDL-c level was also determined. MREt maintained the wet organ and relative weight, as did not produce any harmful changes in kidney, liver, spleen and heart. Significant reduction in percent glycosylation was observed from -37.35 to $-48 \%$ in all test groups. Upto two fold significant $(p<0.0001)$ decrease in atherogenic $(\mathrm{AI})$ and arteriosclerotic $\left(\mathrm{A}_{\mathrm{rt}} \mathrm{I}\right)$ indices, while improvements in atherogenic dyslipidemia ratio (ADR) were also observed in all test groups. Significantly $(p<0.0001)$ lowered level of non-HDL-c and glucose to lipid profile ratios were estimated in all test groups. The obtained results indicated the therapeutic potential of MREt in lowering the risk of atherogenic dyslipidemia, arteriosclerosis and glycosylation in alloxaninduced diabetic mice.
\end{abstract}

\section{INTRODUCTION}

Diabetes is a group of metabolic disorders characterized by persistent hyperglycemia, due to defect in secretion of insulin or its action or both, which results in more than 5\% annual deaths all over the globe (Mahato et al., 2011). Its chronic existence linked with severe dysfunction and damage of various body organs like eyes, kidneys, nerves, heart, blood vessels etc (Clark et al., 2007). One of the frightening complications of diabetes is the promotion of atherogenic risk, as diabetic dyslipidemia is a prominent underlying contributor for the development of cardiovascular diseases (Manjunath et al., 2013). Atherogenic

\footnotetext{
* Corresponding Author
}

Dr. Muhammad Bilal Azmi, Quality Enhancement Cell,

Dow University of Health Science, Karachi-74600, Pakistan.

E-mail: azmibilal@gmail.com, Tel: +92-33-3859826, dyslipidemia is majorly distinguished by elevated concentration of serum triglycerides (TG), total cholesterol, low density lipoprotein cholesterol (LDL-c), small dense low density lipoprotein (sdLDL) particles with low level of high density lipoprotein cholesterol (HDL-c) (Manjunath et al., 2013). Similarly, atherosclerosis is the foremost foundation of arteriosclerosis, which is characterized by hardening and diminishing of arterial wall's elasticity due to the accumulation of atheromatous plaque (Thuong et al., 2009). These circumstances are associated with the over production of free radicals/reactive oxygen species (ROS) in the cellular vicinity leads to the generation of advanced glycated end products (AGEs), which serve as initiating factor for the generation and strengthening of atherogenesis (Rabbani et al., 2011).

Researches elaborated two to three fold greater risk regarding the development of atherosclerosis in patients with diabetes than general population (Kwon et al., 2006). 
Whereas, another study designated diabetes as coronary disease equivalent condition, due to its and silent advancement in cardiovascular disease (Kumar and Singh, 2010). Similarly, 50\% of mortalities in diabetic patients are reported due to different cardiovascular diseases (Mahato et al., 2011). Because of the extraordinary impact, widespread suffering and high mortality ratio due to atherogenic dyslipidemic consequences, demands a new therapeutic strategy.

Worldwide recognized antihypertensive phytomedicine Rauwolfia serpentina Benth (family Apocynaceae) is also wellknown for its diversified ethno-medicinal usefulness which include the cure of snake (venom) bite, gastrointestinal tract disorders, breast cancer, skin problems, etc (Qureshi and Udhani, 2009; Dey and De, 2011). In recent years, its short and long-term antidiabetic activities have been reported in alloxan-induced diabetic mice where it was found to improve the cardioprotective indices (Qureshi et al., 2009; Azmi and Qureshi, 2012a; Azmi and Qureshi, 2012b). Similarly, the use of methanolic root extract of $R$. serpentina also proved its antioxidant and heamatinic potential in alloxan-induced diabetic mice (Azmi and Qureshi, 2013). Therefore, in continuation of past concepts, the present study aimed for the exploration of MREt's preventive role in atherogenic dyslipidemia and arteriosclerotic condition in alloxan-induced type 1 diabetic mice, that has not been reported so far.

\section{MATERIALS AND METHODS}

\section{Plant material and study protocol}

Roots of Rauwolfia serpentina were obtained from authentic dealer of Karachi and identified by specialist of Botany Department, University of Karachi, Karachi-75270, Pakistan. The voucher specimen has been kept in our department (KU/BCH/SAQ/02). Methanolic root extract (MREt) of Rauwolfia serpentina was prepared according to the standardized method, as reported earlier by Azmi and Qureshi in 2012 (Azmi and Qureshi, 2012a). Similarly, experimental albino Wister mice
(25-35 g) of male gender were purchased from the Laboratory Animal House of Dow University of Health Sciences (DUHS), Karachi. Mice were maintained individually in their cages. Room environment for experimental subject was air conditioned to maintain temperature range carefully between $23 \pm 2{ }^{\circ} \mathrm{C}$ with relative humidity in range of about $55 \%$. All experimental mice were provided laboratory standard diet with free access to water $a d$ libitum. In June 2010, Institutional Ethical Review Board (IERB) of DUHS permitted the present study protocol (Authority letter Reference Number: IRB-186/DUHS-10).

\section{Type 1 diabetes induction in experimental mice}

Type 1 diabetes was induced in experimental subjects through single injection (intra-peritoneal) of alloxan monohydrate (150 mg/kg). After 3 days (72 hours approximately) of injection, fasting blood glucose (FBG) levels were monitored through glucometer (Optium Xceed, Diabetes Monitoring system by Abbott) by pricking the tail vein of mice, those showed FBG greater than $190 \mathrm{mg} / \mathrm{dL}$ were included for present study (Azmi and Qureshi, 2012b).

\section{Grouping of experimental mice}

Mice that were overnight (approximately 12 - 14 hours) fasted assigned into total seven (07) groups as described in Figure 1 below. In Positive control group, commercially available antidiabetic medicine 'Glibenclamide' (Doanil) was used which has been purchased from Sanofi-aventis Pakistan Ltd. It was used in concentration of $5 \mathrm{mg} / \mathrm{kg}$. Dimethyl sulphoxide (DMSO) of analytical grade were procured from the distributor of Fisher Scientific (UK). For the purpose of administration of the three test doses of MREt in test animals, its concentration of $0.05 \%$ was used as vehicle. Above treatments were given to their particular groups orally once a day for 14 days consecutively. After the completion of present protocol mice were sacrificed. Whole blood, serum and visceral organs (kidney, liver, spleen and heart) were collected for analytical purpose.

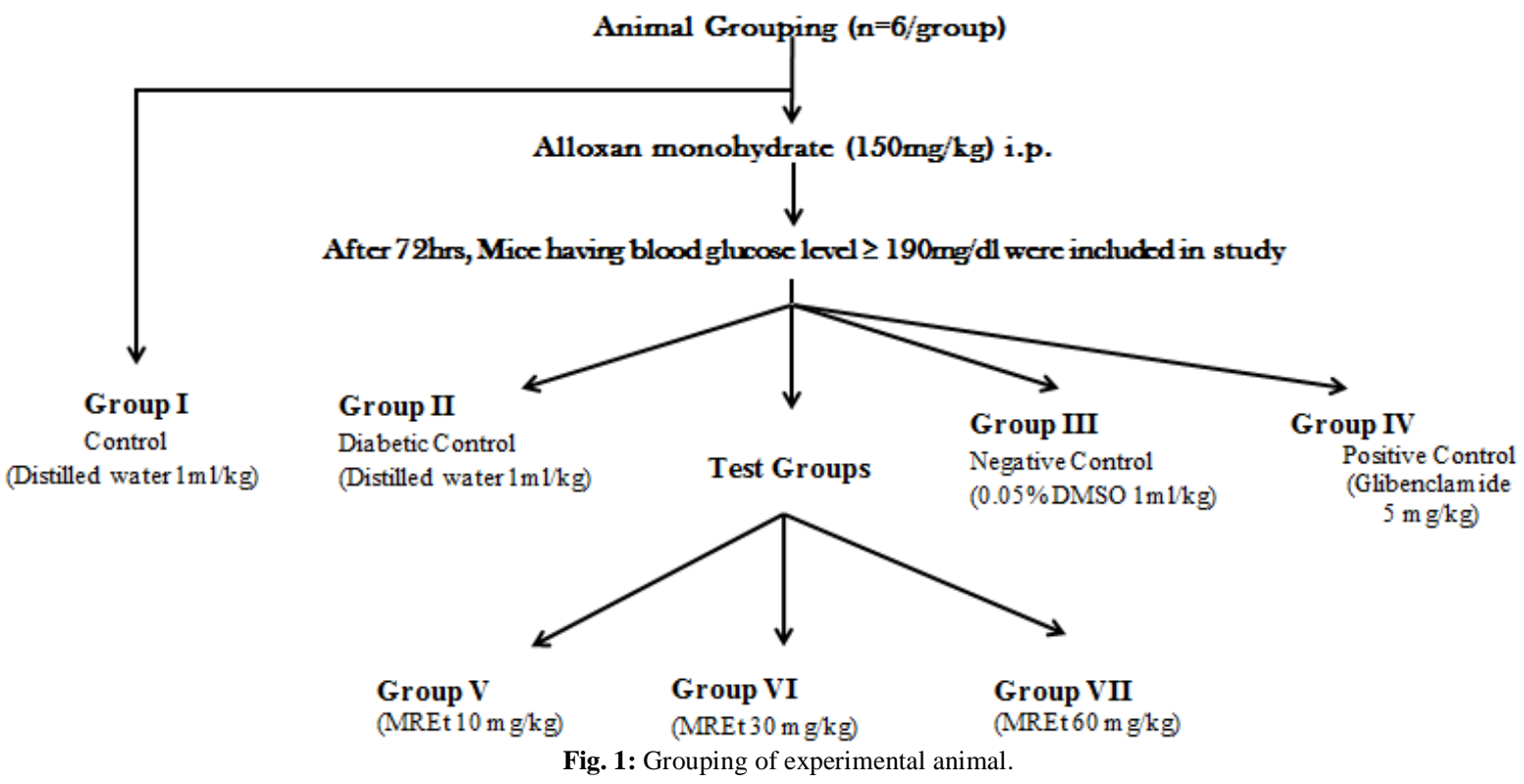


Determination of wet organs weight and their relative weights (percent body weight)

On final day ( $14^{\text {th }}$ day) of animal trial, after sacrificing mice, body organs including heart, kidneys, liver and spleen were dissected out carefully, tabbed on filter paper and their weights were recorded in grams ( $\mathrm{g}$ ) by using digital weighing balance and finally their relative weights (percent body weight) were calculated with the help of following formula (Adaramoye et al., 2012). Relative weight $(\%$ body weight $)=\left(\frac{\text { Wet organ wight }}{\text { Body weight on final day }}\right) \times 100$

\section{Determination of glycosylation indices}

The glycosylation indices were computed through the following formulae with the help of (FBG), total hemoglobin $(\mathrm{Hb})$ and glycosylated hemoglobin $\left(\mathrm{HbA}_{1} \mathrm{c}\right)$ levels on $14^{\text {th }}$ day (Azmi and Qureshi, 2013). $\mathrm{Hb}$ and $\mathrm{HbA}_{1} \mathrm{c}$ were evaluated by Automated Analyzer, Sysmex (XS-1000i) and commercially available Kit (Nycocard Kit, USA) respectively. While, the serum fasting insulin (SFI) levels were determined by cobas e411 analyzer, Hitachi (Roche Diagnostics GmbH, Mannheim, Germany)

$$
\begin{gathered}
\text { Glucose to Hb ratio }=\frac{\mathrm{FBG}\left(\frac{\mathrm{mg}}{\mathrm{dL}}\right)}{\mathrm{Hb}(\%)} \\
\text { Glucose to HbA1c ratio }=\frac{\mathrm{FBG}\left(\frac{\mathrm{mg}}{\mathrm{dL}}\right)}{\mathrm{HbA1c}(\%)} \\
\text { Insulin to } \mathrm{Hb} \text { ratio }=\frac{\mathrm{SFI}(\mathrm{QU} / \mathrm{ml})}{\mathrm{Hb}(\%)} \\
\text { Insulin to HbA1c ratio }=\frac{\mathrm{SFI}(\text { ? U } / \mathrm{ml})}{\mathrm{HbA} 1 \mathrm{c}(\%)} \\
\text { Percent glycosylation change }(\%)=\left(\frac{\mathrm{HbA1c}(\mathrm{X})-\mathrm{HbA1c}(\mathrm{Y})}{\mathrm{HbA1c}(\mathrm{Y})}\right) \times 100
\end{gathered}
$$

Where, HbA1c $(\mathrm{X})=$ glycosylated haemoglobin of Groups I, IV, $\mathrm{V}, \mathrm{VI} \& \mathrm{VII}$ and

HbA1c $(\mathrm{Y})$ = glycosylated haemoglobin of II, \& III groups, compared gradually.

\section{Determination of glucose to lipid markers ratios and non HDL-c}

Glucose to lipid ratios were computed through below mentioned formulae after estimating total cholesterol (T-CHOL), triglycerides (TG), high density lipoprotein-cholesterol (HDL-c) level by commercially available enzymatic assay kits (Randox, United Kingdom). Whereas low density lipoprotein-cholesterol (LDL-c), very low density lipoprotein-cholesterol (VLDL-c) and Non-HDL-c were calculated by authentic formulae (Friedewald et al., 1972; Virani, 2011).

$$
\begin{aligned}
\text { Glucose to } \mathrm{T}-\mathrm{CHOL} \text { ratio } & =\frac{\mathrm{FBG}}{\mathrm{T}-\mathrm{CHOL}} \\
\text { Glucose to TG ratio } & =\frac{\mathrm{FBG}}{\mathrm{TG}} \\
\text { Glucose to } \mathrm{LDL}-\mathrm{c} \text { ratio } & =\frac{\mathrm{FBG}}{\mathrm{LDL}-\mathrm{c}} \\
\text { Glucose to } \mathrm{VLDL}-\mathrm{c} \text { ratio } & =\frac{\mathrm{FBG}}{\mathrm{VLDL}-\mathrm{c}} \\
\text { Non-HDL-C }(\mathrm{mg} / \mathrm{dL}) & =\mathrm{T}-\mathrm{CHOL}-\mathrm{HDL}-\mathrm{c}
\end{aligned}
$$

\section{Determination of Atherogenic Index (AI), Atherogenic Dyslipidemia Ratio (ADR) and Arteriosclerosis Index $\left(\mathrm{A}_{\mathrm{rt}} \mathrm{I}\right)$}

Atherogenic Index (AI), Atherogenic Dyslipidemia ratio $(\mathrm{ADR})$ and Arteriosclerosis Index $\left(\mathrm{A}_{\mathrm{rt}} \mathrm{I}\right)$ were calculated as described by Adeneye and Olagunju, 2009, Hermans et al., 2012, and Zargar et al., 2014 respectively.

$\mathrm{AI}=\frac{\mathrm{LDL}-\mathrm{c}}{\mathrm{HDL}-\mathrm{c}}$

$\mathrm{ADR}=[\log (\mathrm{TG}) / \mathrm{HDL}-\mathrm{C}]$

Art I $=\mathrm{T}-\mathrm{CHOL}-\left(\frac{\mathrm{HDL}-\mathrm{c}}{\mathrm{HDL}-\mathrm{c}}\right)$

\section{Statistical analysis}

Results of the present study are expressed as Mean \pm SD (Standard deviation). The data were analyzed with statistical package for social science (SPSS version 18) by using one-way ANOVA followed by LSD (least significant difference) test at $p<$ 0.05 . The differences were considered significant at $p<0.05, p<$ $0.01, p<0.001$ and $p<0.0001$ when compared with respective controls.

\section{RESULTS}

\section{Effect of MREt on relative organ body weights}

Consecutive long-term administration of MREt in alloxan-induced diabetic mice didn't produce any lethal change i.e., increment or decrement in relative organ body weights. All the three dose group of MREt $(10,30 \& 60 \mathrm{mg} / \mathrm{kg})$ were found safe for kidney, liver, spleen and heart tissues when compared with normal control group (Table 1).

\section{Effect of MREt treatment on glycosylation indices of alloxan- induced diabetic mice}

All three doses of MREt $(10,30 \& 60 \mathrm{mg} / \mathrm{kg})$ produce significant $(* * * p<0.0001)$ decrement in glucose to $\mathrm{Hb}(\mathrm{Glu} / \mathrm{Hb})$ ratio which depicts the increment in $\mathrm{Hb}$ levels and decrement in glucose quantity after the repeated administration of MREt. However, the reverse was observed in diabetic and negative control groups.

Interestingly, the high ratios of glucose to $\mathrm{HbA} 1 \mathrm{c}$ in diabetic and negative control groups witness the glycosylation after the administration of alloxan in mice. Whereas, all the three doses of MREt $(10,30 \& 60 \mathrm{mg} / \mathrm{kg})$ significantly $(* p<0.05, * * p$ $<0.01 \& * * * p<0.0001)$ showed the lowering of glucose to $\mathrm{HbA1c}$ ratio that reflects the betterment in glucose linked glycosylation pattern after MREt used (Table 2).

Contrary, insulin to $\mathrm{Hb}$ ratio in MREt treated $(10,30 \&$ $60 \mathrm{mg} / \mathrm{kg}$ ) test groups also confirm the improvement $(* * * p<$ $0.0001)$ in both of these markers, whereas a good and similar impact of same index was also observed in positive control groups. Beside this, significant $(* * * p<0.0001)$ improvement in insulin to $\mathrm{HbA1c}$ ratio, further validates the lowering of (HbA1c) and improvement in insulin level after the consecutive administration of MREt in test groups. Interestingly both of these indices of diabetic control groups witness the decrease in glycosylation pattern (Table 2). 
Table 1: Effect of MREt on Wet and Relative Organ Body Weights of Alloxan-Induced Diabetic Mice.

\begin{tabular}{|c|c|c|c|c|c|c|c|c|}
\hline \multirow{2}{*}{ Groups } & \multicolumn{4}{|c|}{ Wet organ weight (g) } & \multicolumn{4}{|c|}{ Relative Weight (\% body weight) } \\
\hline & Kidney & Liver & Spleen & Heart & Kidney & Liver & Spleen & Heart \\
\hline Group I & $0.40 \pm 0.04$ & $1.44 \pm .05$ & $0.11 \pm 0.02$ & $0.10 \pm 0.01$ & $1.29 \pm 0.14$ & $4.52 \pm 0.28$ & $0.35 \pm 0.07$ & $0.32 \pm 0.04$ \\
\hline Group II & $0.40 \pm 0.05$ & $1.44 \pm 0.07$ & $0.11 \pm 0.03$ & $0.11 \pm 0.02$ & $1.56 \pm 0.31$ & $5.58 \pm 0.76$ & $0.42 \pm 0.13$ & $0.40 \pm 0.05$ \\
\hline Group III & $0.39 \pm 0.03$ & $1.46 \pm 0.08$ & $0.11 \pm 0.04$ & $0.10 \pm 0.01$ & $1.45 \pm 0.15$ & $5.46 \pm 0.52$ & $0.39 \pm 0.13$ & $0.35 \pm 0.03$ \\
\hline Group IV & $0.40 \pm 0.05$ & $1.41 \pm 0.07$ & $0.10 \pm 0.02$ & $0.10 \pm 0.02$ & $1.25 \pm 0.15^{* \mathrm{a}}$ & $4.41 \pm 0.12^{* a b}$ & $0.31 \pm 0.07$ & $0.32 \pm 0.05^{*_{\mathrm{a}}}$ \\
\hline Group V & $0.40 \pm 0.03$ & $1.40 \pm 0.12$ & $0.10 \pm 0.02$ & $0.11 \pm 0.02$ & $1.38 \pm 0.13$ & $4.84 \pm 0.41$ & $0.35 \pm 0.10$ & $0.37 \pm 0.03$ \\
\hline Group VI & $0.38 \pm 0.02$ & $1.47 \pm 0.04$ & $0.12 \pm 0.02$ & $0.10 \pm 0.03$ & $1.44 \pm 0.17$ & $5.56 \pm 0.77$ & $0.44 \pm 0.12$ & $0.36 \pm 0.09$ \\
\hline Group VII & $0.40 \pm 0.03$ & $1.43 \pm 0.07$ & $0.10 \pm 0.02$ & $0.11 \pm 0.01$ & $1.52 \pm 0.27$ & $5.42 \pm 0.61$ & $0.38 \pm 0.09$ & $0.40 \pm 0.03$ \\
\hline
\end{tabular}

Values are expressed as mean \pm SD $(n=6) .{ }^{*} p<0.05$ when compared with respective group II (a) and III (b)

Table 2: Effect of MREt on Glycosylation Indices of Alloxan-Induced Diabetic Mice.

\begin{tabular}{|c|c|c|c|c|}
\hline Groups & FBG to Hb ratio & FBG to HbA1c ratio & SFI to Hb ratio & SFI to HbA1c ratio \\
\hline I & $7.22 \pm 0.66$ & $16.14 \pm 2.55$ & $0.03 \pm 0.002$ & $0.07 \pm 0.008$ \\
\hline II & $23.99 \pm 3.72$ & $20.84 \pm 3.32$ & $0.02 \pm 0.005$ & $0.02 \pm 0.00$ \\
\hline III & $25.12 \pm 3.92$ & $23.74 \pm 2.71$ & $0.02 \pm 0.005$ & $0.02 \pm 0.005$ \\
\hline IV & $9.61 \pm 2.31^{* * * a b}$ & $17.80 \pm 6.22$ & $0.05 \pm 0.00^{* * * * a b}$ & $0.09 \pm 0.02^{* * * a b}$ \\
\hline $\mathrm{V}$ & $7.42 \pm 0.90^{* * * a b}$ & $13.53 \pm 0.76^{* * a^{* * * b}}$ & $0.02 \pm 0.00$ & $0.05 \pm 0.006^{* * * a b}$ \\
\hline VI & $8.08 \pm 1.86^{* * * a b}$ & $14.24 \pm 3.62^{*_{\mathrm{a} * * \mathrm{~b}}}$ & $0.04 \pm 0.005^{* * * a b}$ & $0.08 \pm 0.00^{* * * a b}$ \\
\hline VII & $7.70 \pm 1.04^{* * * a b}$ & $15.47 \pm 2.66^{* \mathrm{a}^{* * \mathrm{~b}}}$ & $0.03 \pm 0.005^{* * * a b}$ & $0.08 \pm 0.005^{* * * a b}$ \\
\hline
\end{tabular}

Results are expressed as mean \pm SD $(n=6) . * p<0.05, * * p<0.01$, and $* * * p<0.0001$, when compared with respective group II (a) and III (b).

Table 3: Effect of MREt on AI, ADR and Art I in Alloxan-Induced Diabetic Mice.

\begin{tabular}{cccr}
\hline Groups & AI & ADR & Art I \\
\hline I & $1.39 \pm 0.59$ & $0.04 \pm 0.02$ & $140.45 \pm 25.75$ \\
II & $3.98 \pm 2.42$ & $0.04 \pm 0.02$ & $259.48 \pm 20.36$ \\
III & $4.32 \pm 2.46$ & $0.05 \pm 0.02$ & $253.12 \pm 29.85$ \\
IV & $4.26 \pm 1.95$ & $0.06 \pm 0.02$ & $177.40 \pm 31.90^{* * * a * * b}$ \\
V & $2.36 \pm 0.54$ & $0.05 \pm 0.020$ & $145.21 \pm 20.02^{* * * a b}$ \\
VI & $2.14 \pm 1.81$ & $0.06 \pm 0.02$ & $128.32 \pm 38.70^{* * * a b}$ \\
VII & $2.04 \pm 0.73$ & $0.05 \pm 0.02$ & $137.37 \pm 18.49^{* * * a b}$ \\
\hline
\end{tabular}

Results are expressed as mean \pm SD $(n=6) . * p<0.05, * * p<0.01$, and ***p $<0.0001$, when compared with respective group II (a) and III (b).

Table 4: Effect of MREt on FBG to T-CHOL, TG and lipoproteins (LDL-c \& VLDL-c) Indices in Alloxan-Induced Diabetic Mice.

\begin{tabular}{|c|c|c|c|c|}
\hline Groups & FBG to T-CHOL ratio & FBG to TG ratio & FBG to LDL-c ratio & FBG to VLDL-c ratio \\
\hline I & $0.71 \pm 0.18$ & $0.66 \pm 0.09$ & $1.61 \pm 0.74$ & $3.29 \pm 0.43$ \\
\hline II & $0.95 \pm 0.22$ & $1.36 \pm 0.41$ & $1.46 \pm 0.46$ & $7.6 \pm 1.21$ \\
\hline III & $1.01 \pm 0.09$ & $1.68 \pm 0.78$ & $1.58 \pm 0.11$ & $7.82 \pm 2.68$ \\
\hline IV & $0.68 \pm 0.26^{* \mathrm{~b}}$ & $0.97 \pm 0.26$ & $0.77 \pm 0.89$ & $4.83 \pm 1.31^{* * a b}$ \\
\hline $\mathrm{V}$ & $0.63 \pm 0.19^{* a b}$ & $0.73 \pm 0.05^{* \mathrm{a}^{* * \mathrm{~b}}}$ & $1.58 \pm 0.80$ & $3.75 \pm 0.20^{* * a * * b b}$ \\
\hline VI & $0.84 \pm 0.25$ & $0.73 \pm 0.27^{* \mathrm{a}^{* * \mathrm{~b}}}$ & $2.39 \pm 1.40$ & $3.67 \pm 1.36^{* \mathrm{a}^{* * * \mathrm{~b}}}$ \\
\hline VII & $0.72 \pm 0.16^{* b}$ & $0.73 \pm 0.18^{* \mathrm{a}^{* * \mathrm{~b}}}$ & $1.42 \pm 0.46$ & $3.62 \pm 0.90^{* * a * * b b}$ \\
\hline
\end{tabular}

Results are expressed as mean \pm SD $(n=6)$. $* p<0.05, * * p<0.01$, and $* * * p<0.0001$, when compared with respective group II (a) and III (b).

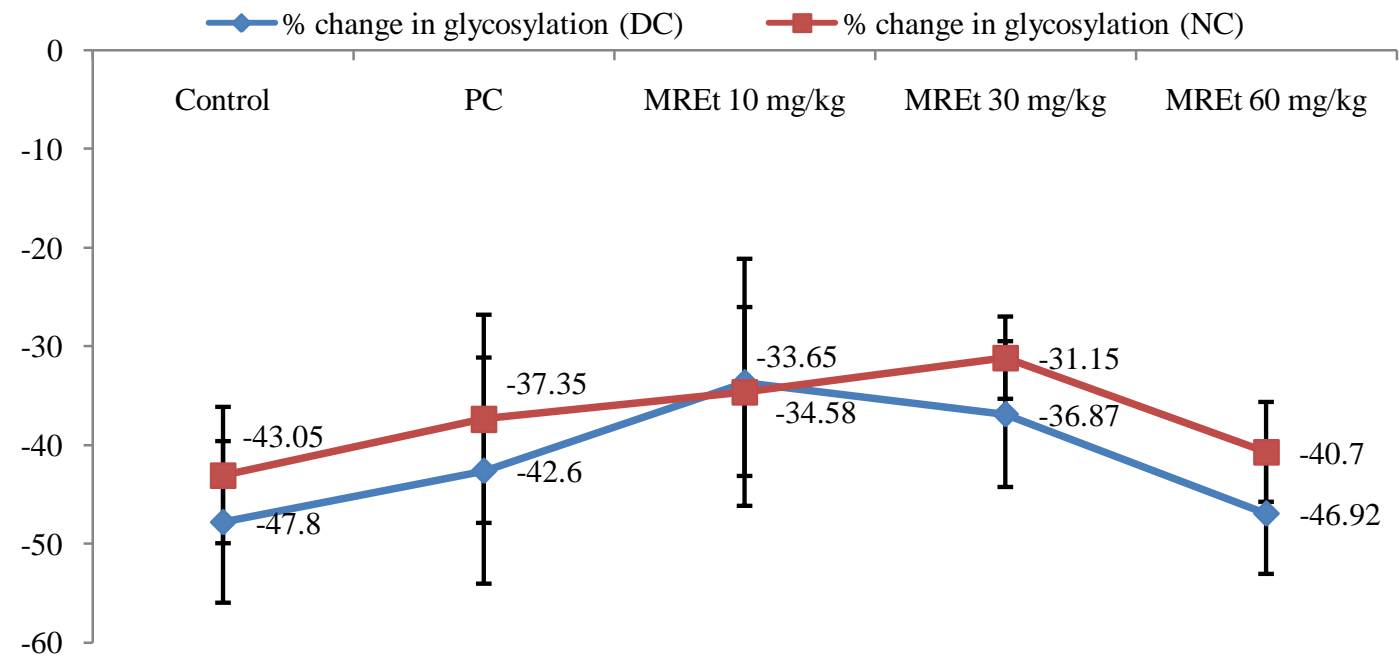

Fig. 2: Effect of MREt on percent (\%) change in glycosylation in Alloxan-Induced Diabetic Mice $\mathrm{DC}=$ when compared with Diabetic control, $\mathrm{NC}=$ when compared with Negative control groups. Negative sign indicates percent reduction. 
口Non-HDL-c (mg/dL) level

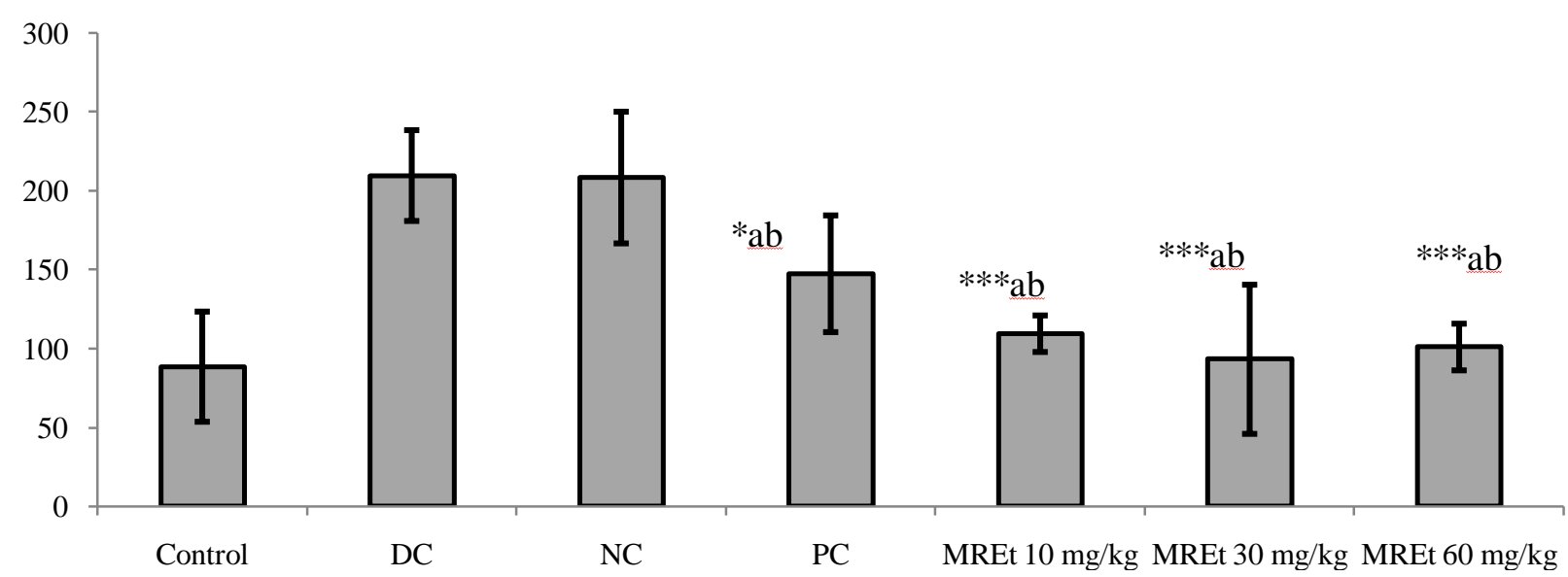

Fig. 3: Effect of MREt on Non-HDL-c level in Alloxan-Induced Diabetic Mice

Results are expressed as mean $\pm \mathrm{SD}(n=6) .{ }^{*} \mathrm{p}<0.05$ and $* * * p<0.0001$, when compared with respective group II (a) and III (b).

Effect of MREt treatments on Non-HDL-c, Atherogenic Index (AI), Atherogenic Dyslipidemia ratio (ADR) and

Arteriosclerosis Index $\left(A_{\mathrm{rt}} \mathrm{I}\right)$ of alloxan-induced diabetic mice

The estimation of non-HDL-c in all groups showed a very clear picture in this regard as a prominent increment was observed in diabetic and negative controls. While, the similar fraction was significantly $(* * * p<0.0001)$ decreased in all the test doses of MREt. Positive control also showed same decrement (Fig. 3). After the repeated administration of MREt $(10,30 \& 60$ $\mathrm{mg} / \mathrm{kg}$ ), a gradual decrement in AI was also observed while it's almost double magnitude was estimated in diabetic controls. However, estimated ADR also reflects the same scenario hence it established the effectiveness of MREt in this regard. Additionally, $\mathrm{A}_{\mathrm{rt}} \mathrm{I}$ was also significantly (***p $\left.<0.0001\right)$ decreased in all three MREt test groups while prominent increment was noticed in diabetic control groups (Table 3 ).

Effect of MREt treatments on glucose to cholesterol (Glu/TCHOL), Triglycerides (Glu/ TG) and lipoproteins (Glu/ LDLc) and (Glu/ VLDL-c) indices of alloxan-induced diabetic mice

MREt test doses reduces the Glu/T-CHOL ratio upto significant $\left({ }^{*} p<0.05\right)$ extent while Glu/TG ratio also confirms the same impact in significant $(* p<0.05, \& * * p<0.01)$ manner (Table 4). Glu/LDL-c ratio also verifies the lowering of blood glucose levels and LDL-c contents in test groups. Significant $(* * p$ $<0.01 \& * * * p<0.0001)$ decrement in gradual manner was also noticed in MREt dose treated test groups as compared to diabetic control groups, which showed almost double figures of this index (Table 4).

Effect of MREt treatments on percent change in glyocsylation of alloxan-induced diabetic mice

A prominent reduction in glycosylation pattern was observed as $-33.65 \%,-36.87 \%,-46.92,-42.6 \% \&-47.8 \%$ respectively in three MREt treated test, positive control and normal control groups when compared with diabetic control group. However, when compared with negative control group, percent reduction in glycosylation $-34.58 \%,-31.15 \%,-40.70 \%,-43.05 \%$ \& $-37.35 \%$ were observed in same groups (Fig 2).

\section{DISCUSSION}

Today in spite of the accessibility of pharmaceutical formulations, science of phytomedines or natural products gaining importance in providing benefits to mankind. It has been reported that more than $80 \%$ population from developing and developed world utilizes these benefits in curing various disease (Tiwari, 2008). The productive effects are majorly due to the presence of various secondary metabolites that enhance the versatile ethnopharmacological properties of medicinal plants with almost negligible side effects (Pitchai et al., 2010).

In the similar manner, well-known Rauwolfia serpentina is popular for its anti-hypertensive property. Beside this, it has also been reported in the management of many neurological disorders include schizophrenia, anxiety, insanity, etc (Dey and De, 2011). This pharmacological action is due to the presence of variety of indole alkaloids like ajamlicine, ajmaline, reserpine, etc, that have been reported earlier depletes the level of catecholamines in the central and peripheral nervous system (Azmi and Qureshi, 2012a). From 2009 onwards, well established researches reported the use of methnolic root extracts of $R$. serpentina in normoglycemic as well as alloxan-induced diabetic animal models (Qureshi et al., 2009; Azmi and Qureshi, 2012b). Similarly, studies like acute toxicity, oral glucose tolerance and phytochemical analysis also provide a smooth foundation for its modern use (Azmi and Qureshi, 2012a; Azmi and Qureshi, 2012b). On the other hand, advanced computational studies on alkaloids of $R$. serpentina highlighted their role as insulin receptor activators and aldose 
reductase inhibitors, which also strengthen the antidiabetic activity of this plant (Ganugapati et al., 2012; Pathania et al., 2013). Now present effort evaluated the use of MREt of $R$. serpentina in reducing the risk of atherogenic dyslipidemia and arteriosclerosis in alloxan-induced diabetic mice.

Many researches frequently used relative body and organ weights, due to their helpful role in assessing the situation when these are disturbed or affected due to chemicals or toxins (Peters and Boyd, 1966; Pfeiffer, 1968). After the administration of MREt in alloxan-induced diabetic mice for consecutive 14 days, the wet organ weight, post treatment body weight and relative weight (\% body weight) were measured. Results showed their weight range as same as observed in normal control group that indicated the non-deleterious and non-hazardous impact of MREt (Table 1). Extraordinary increment or decrements in relative weights have long been used as an indicator of drug induced effects on visceral organs (Michael et al., 2007), like liver is the primary organ, actively involved in the detoxification of almost all types of drugs and substances (Azmi and Qureshi, 2012b). Therefore, the liver function should not be affected after taking any orally administrated medicine or plant extract (Azmi and Qureshi, 2013). Similarly, kidney weight is also serves as a sensitive tool to assess its anatomy and excretory property which could be affected either due to malfunctioning liver cells (due enzyme induction) or lipidosis or biochemical alteration due to the action of any chemical or drug (Michael et al., 2007). While, heart weights have limited inference due to its size and physiologic specificity, but it was often dependent with any increment or decrement in weight of body (Michael et al., 2007). Likewise, deleterious change or increment in relative weight of spleen may refer as sensitive sign for immune associated toxicities and other cellular damages (Michael et al., 2007).

Diabetes is a life-threatening metabolic disorder which has been characteristically associated with an increase in glycosylated or glycated hemoglobin (HbA1c). In diabetes, excess blood glucose non-enzymatically reacts with $\mathrm{Hb}$, thereby converting into $\mathrm{HbA}_{1 \mathrm{c}}$ and increasing its quantity more than $6 \%$ which severely affects the normal physiologic functions of many biological proteins in body (Azmi and Qureshi, 2013; Azmi and Qureshi, 2012b). Prolonged hyperglycemic existence in diabetes participates in the formation and accumulation of advance glycated end products (AGES) which correlates with various severities like generation of free radical or reactive oxygen species (ROS), renal complications, formation of cataract and development atherosclerosis etc (Lorenzi, 2007; Rabbani et al., 2011). In present work, all the set glycosylation indices were significantly $\left({ }^{*} p<0.05, * * p<0.01 \&{ }^{* * *} p<0.0001\right)$ improved in all treated groups of MREt as $-37.35 \%$ to $-48 \%$ glycosylation reductions were observed in these groups. It is one of the noteworthy aspects of root extract of R.serpentina that it prevents the glycosylation of hemoglobin, which could in turn inhibit the formation of AGES and protects the body functions. Interestingly it was also validated by observing the decrease in percent glycosylation change (Fig. 2). Quantifying non-HDL-c is often used as an indicator of quantity of cholesterol carried within all lipoproteins and served as an atherogenic identifying marker (Virani, 2011). Non-HDL-c actually reflects the presences of atherogenic lipoproteins including VLDL-c, IDL-c (intermediate density lipoprotein) and small dense LDL (sdLDL) particles in patients (Virani, 2011). In the present study, the measurement of non-HDL-c proved the beneficial effect of MREt, as its treatment significantly $(* * * p<$ 0.0001) maintained the same fraction in a very ideal range less than $130 \mathrm{mg} / \mathrm{dL}$ (Fig. 3). Contrary, the diabetic controls verified the presence of atherogenic risk by showing the same parameter more than $200 \mathrm{mg} / \mathrm{dL}$ which is the clear picture of a very high risk. Atherogenicity is related with the elevated levels of T-CHOL, TG, LDL-c, VLDL-c and sdLDL particles which are derived from TG rich VLDL (Manjunath et al., 2013). Both LDL-c and sdLDL acts as transporting vehicles for cholesterol and TG respectively and transported them to arteries or peripheral tissues. Of which, sdLDL-c is the most efficient as it is smaller than other lipoproteins so it can easily move to the peripheral tissues where macrophages can engulf them and through oxidative transformation turn into foam cells which later deposited as plaque on walls of coronary arteries and referred as atheroma (Qureshi $e t$ al., 2010; Rabbani et al., 2011). Through MREt treatments, almost double decrement in atherogenic index was noticed in all test groups as compared to diabetic controls (Table 2). This reflects the potential of MREt of R.serpentina in lowering the formation of atheroma plaque in arterial walls. Similarly, the improvement in arteriosclerosis index also confirmed this beneficial aspect of MREt in the present study

Atherogenic dyslipidemic ratio (ADR) is another useful tool to assess the future risk of cardiovascular diseases (Hermans and Fruchart, 2010) and it is also associated with decreased insulin secretion with poor glycemic control, dysfunctioning of $\beta$-cell, and greater incidence of microangiopathies (Hermans et al., 2012). Through improved ADR, the effectiveness of MREt treatment was also confirmed by observing its reducing effect on TG and elevating effect on HDL-c (Table 3). Similarly, all estimated glucose to lipid ratios in MREt treated test groups were found improved which assured the possible use MREt in treatment of diabetes and diabetic dyslipidemia.

\section{CONCLUSION}

The obtained findings indicated the therapeutic potential of MREt in lowering the risk of atherogenic dyslipidemia and arteriosclerosis by improving the glycosylation and glucose to lipid ratios in alloxan-induced diabetic mice.

\section{REFERENCES}

Adaramoye O, Amanlou M, Habibi-Rezaei M, Pasalar P, Ali MM . Methanolic extract of African mistletoe (Viscum album) improves carbohydrate metabolism and hyperlipidemia in streptozotocin-induced diabetic rats. Asian Pac J Trop Med, 2011; 5:427-433.

Adeneye AA, Olagunju JA. Preliminary hypoglycemic and hypolipidemic activities of the aqueous seed extract of Carica papaya Linn. in Wistar rats. Biol Med, 2009; 1:1-10. 
Azmi MB, Qureshi SA. Methanolic root extract of Rauwolfia serpentina Benth improves the glucose tolerance in Wister mice. J Food Drug Anal, 2012a; 20:54-58.

Azmi MB, Qureshi SA. Rauwolfia serpentina ameliorates hyperglycemic, haematinic and antioxidant status in alloxan- induced diabetic mice. J App Pharm Sci, 2013; 3:136-141.

Azmi MB, Qureshi SA. Methanolic root extract of Rauwolfia serpentina Benth improves the glycemic, antiatherogenic, and cardioprotective indices in alloxan-induced diabetic mice. Adv Pharmacol Sci, 2012b; article ID 376429. 11pages.

Clark NG, Fox KM, Grandy S. Symptoms of diabetes and their association with the risk and presence of diabetes. Diabetes Care, 2007; 30:2868-2873

Dey A, De JN. Ethnobotanical aspects of Rauwolfia serpentina (L). Benth. ex Kurz. in India, Nepal and Bangladesh. J Med Plant Res, 2011; 5:144-150.

Friedewald WT, Levy RI, Fredrickson DS. Estimation of the concentration of low-density lipoprotein cholesterol in plasma without use of the preparative ultracentrifuge.Clin Chem, $1972 ; 18: 499-502$

Ganugapati J, Baldwa A, Lalani S. Docking studies of Rauwolfia serpentina alkaloids as insulin receptor activators. Int $\mathrm{J}$ Computer Applicat, 2012; 43:32-37.

Hermans MP, Fruchart JC. Reducing residual vascular risk in patients with atherogenic dyslipidaemia: where do we go from here? Clin Lipidology, 2010; 5:811-826.

Hermans M P, Ahn SA, Rousseau MF. The atherogenic dyslipidemia ratio $[\log (\mathrm{TG}) / \mathrm{HDL}-\mathrm{C}]$ is associated with residual vascular risk, beta-cell function loss and microangiopathy in type 2 diabetes females. Lipids Health Dis, 2012; 11:132

Kumar A, Singh V. Atherogenic dyslipidemia and diabetes mellitus: what's new in the management arena?. Vasc Health Risk Manag, 2010; 6:665-669.

Kwon SW, Yoon SJ, Kang TS, Kwon HM, Kim JH, Rhee J, et al. Significance of small dense low density lipoprotein as a risk factor for coronary artery disease and acute coronary syndrome. Yonsei Med J 2006; 47:405-14

Lorenzi M. The polyol pathway as a mechanism for diabetic retinopathy: attractive, elusive, and resilient. Exp Diabetes Res, 2007, Article ID 61038: 10 pages

Mahato RV, Gyawali P, Raut PP, Regmi P, Singh KP, Pandeya DR, Gyawali P. Association between glycaemic control and serum lipid profile in type 2 diabetic patients: Glycated haemoglobin as a dual biomarker. Biomed Res, 2011; 22:375-380.

Manjunath CN, Rawal JR, Irani PM, Madhu K. Atherogenic dyslipidemia. Indian J Endocr Metab. 2013; 17:969-76.

Michael B, Yano B, Sellers RS, Perry R, Morton D, Roome N, Johnson JK, Schafer K. Evaluation of organ weights for rodent and nonrodent toxicity studies: A review of Regulatory Guidelines and a Survey of Current Practices. Toxicologic Pathology. 2007; 35:742-750.
Pathania S, Randhawa V, Bagler G. Prospecting for novel plant-derived molecules of rauvolfia serpentina as inhibitors of aldose reductase, a potent drug target for diabetes and its complications. PLoS ONE, 2013; 8:e61327.

Peters J M, Boyd EM. Organ weights and water levels of the rat following reduced food intake. J Nutr, 1966; 90:354-60.

Pfeiffer CJ. A mathematical evaluation of the thymic weight parameter. Toxicol Appl Pharmacol. 1968; 13:220-7.

Pitchai D, Manikkam R, Rajendran SR, Pitchai G. Database on pharmacophore analysis of active principles, from medicinal plants. Bioinformation. $2010 ; 5: 43-45$.

Qureshi SA, Nawaz A, Udani SK, Azmi B. Hypoglycaemic and hypolipidemic activities of Rauwolfia serpentina in alloxan - induced diabetic rats. Int J Pharmacol. 2009; 5:323-326.

Qureshi SA, Udani SK. Hypolipidaemic activity of Rauwolfia serpentina Benth. Pak J Nutr 2009; 8:1103-1106.

Qureshi SA, Kamran M, Asad M, Zia A, Lateef T, Azmi MB. A preliminary study of Santalum album on serum lipids and enzymes. Global J Pharmacol. 2010; 4:71-74.

Rabbani N, Godfrey L, Xue M, Shaheen F, Geoffrion M, Milne R, Thornalley PJ. Glycation of LDL by Methylglyoxal Increases Arterial Atherogenicity A Possible Contributor to Increased Risk of Cardiovascular Disease in Diabetes. Diabetes, 2011; 60:1973-1980.

Thuong PT, Pokharel YR, Lee MY, Kim SK, Bae K, Su ND, Oh WK, Kang KW. Dual Anti-oxidative Effects of Fraxetin Isolated from Fraxinus Rhinchophylla. Biol Pharm Bull 2009; 32:1527-1532.

Tiwari S. Plant: A rich source of herbal medicine. J Nat Prod. 2008; 1:27-35.

Virani SS. Non-HDL Cholesterol as a Metric of Good Quality of Care Opportunities and Challenges. Tex Heart Inst J, 2011; 38:160-162. Zargar BA, Masoodi MH, Ahmed B, Ganie SA. Antihyperlipidemic and Antioxidant Potential of Paeonia emodi Royle against High-Fat Diet Induced Oxidative Stress. ISRN Pharmacol, Volume 2014, Article ID 182362, 7 pages.

\section{How to cite this article:}

Muhammad Bilal Azmi, Shamim A Qureshi, Sumera Rais, Saleha Sultana. Methanolic Root Extract of Rauwolfia serpentina Lowers Atherogenic Dyslipidemia, Arteriosclerosis and Glycosylation Indices in Type 1 Diabetic Mice. J App Pharm Sci, 2015; 5 (08): 061-067. 